\title{
Description Logics with Concrete Domains and General Concept Inclusions Revisited
}

\author{
Franz Baader ${ }^{(凶)}$ and Jakub Rydval \\ Institute of Theoretical Computer Science, TU Dresden, Dresden, Germany \\ $\{$ franz.baader, jakub.rydval\}@tu-dresden.de
}

\begin{abstract}
Concrete domains have been introduced in the area of Description Logic to enable reference to concrete objects (such as numbers) and predefined predicates on these objects (such as numerical comparisons) when defining concepts. Unfortunately, in the presence of general concept inclusions (GCIs), which are supported by all modern DL systems, adding concrete domains may easily lead to undecidability. One contribution of this paper is to strengthen the existing undecidability results further by showing that concrete domains even weaker than the ones considered in the previous proofs may cause undecidability. To regain decidability in the presence of GCIs, quite strong restrictions, in sum called $\omega$-admissibility, need to be imposed on the concrete domain. On the one hand, we generalize the notion of $\omega$-admissibility from concrete domains with only binary predicates to concrete domains with predicates of arbitrary arity. On the other hand, we relate $\omega$-admissibility to well-known notions from model theory. In particular, we show that finitely bounded, homogeneous structures yield $\omega$-admissible concrete domains. This allows us to show $\omega$-admissibility of concrete domains using existing results from model theory.
\end{abstract}

Keywords: Description logic $\cdot$ Concrete domains $\cdot$ GCIs · $\omega$-admissibility $\cdot$ Homogeneity $\cdot$ Finite boundedness $\cdot$ Decidability $\cdot$ Constraint satisfaction

\section{Introduction}

Description Logics (DLs) [3,7] are a well-investigated family of logic-based knowledge representation languages, which are frequently used to formalize ontologies for application domains such as the Semantic Web [27] or biology and medicine [26]. To define the important notions of such an application domain as formal concepts, DLs state necessary and sufficient conditions for an individual to belong to a concept. These conditions can be Boolean combinations of atomic properties required for the individual (expressed by concept names) or

Supported by DFG GRK 1763 (QuantLA) and TRR 248 (cpec, grant 389792660).

(C) Springer Nature Switzerland AG 2020

N. Peltier and V. Sofronie-Stokkermans (Eds.): IJCAR 2020, LNAI 12166, pp. 413-431, 2020.

https://doi.org/10.1007/978-3-030-51074-9_24 
properties that refer to relationships with other individuals and their properties (expressed as role restrictions). For example, the concept of a father that has only daughters can be formalized by the concept description

$$
C:=\neg \text { Female } \sqcap \exists \text { child.Human } \sqcap \forall \text { child.Female, }
$$

which uses the concept names Female and Human and the role name child as well as the concept constructors negation $(\neg)$, conjunction $(\sqcap)$, existential restriction $(\exists r . D)$, and value restriction $(\forall r . D)$. The GCIs

\section{Human $\sqsubseteq \forall$ child.Human and $\exists$ child.Human $\sqsubseteq$ Human}

say that humans have only human children, and they are the only ones that can have human children.

DL systems provide their users with reasoning services that allow them to derive implicit knowledge from the explicitly represented one. In our example, the above GCIs imply that elements of our concept $C$ also belong to the concept $D:=$ Human $\sqcap \forall$ child.Human, i.e., $C$ is subsumed by $D$ w.r.t. these GCIs. A specific DL is determined by which kind of concept constructors are available. A major goal of DL research was and still is to find a good compromise between expressiveness and the complexity of reasoning, i.e., to locate DLs that are expressive enough for interesting applications, but still have inference problems (like subsumption) that are decidable and preferably of a low complexity. For the DL $\mathcal{A L C}$, in which all the concept descriptions used in the above example can be expressed, the subsumption problem w.r.t. GCIs is ExpTime-complete [7].

Classical DLs like $\mathcal{A L C}$ cannot refer to concrete objects and predefined relations over these objects when defining concepts. For example, a constraint stating that parents are strictly older than their children cannot be expressed in $\mathcal{A L C}$. To overcome this deficit, a scheme for integrating certain well-behaved concrete domains, called admissible, into $\mathcal{A L C}$ was introduced in [4], and it was shown that this integration leaves the relevant inference problems (such as subsumption) decidable. Basically, admissibility requires that the set of predicates of the concrete domain is closed under negation and that the constraint satisfaction problem (CSP) for the concrete domain is decidable. However, in this setting, GCIs were not considered since they were not a standard feature of DLs then, ${ }^{1}$ though a combination of concrete domains and GCIs would be useful in many applications. For example, using the syntax employed in [33] and also in the present paper, the above constraint regarding the age of parents and their children could be expressed by the GCI Human $\sqcap \exists$ childage, age.[>] $\sqsubseteq \perp$, which says that there cannot be a human whose age is smaller than the age of one of his or her children. Here $\perp$ is the bottom concept, which is always interpreted as the empty set, age is a concrete feature that maps from the abstract domain populating concepts into the concrete domain of natural numbers, and $>$ is the usual greater predicate on the natural numbers.

\footnotetext{
${ }^{1}$ Actually, they were introduced (with a different name) at about the same time as concrete domains $[2,38]$.
} 
A first indication that concrete domains might be harmful for decidability was given in [6], where it was shown that adding transitive closure of roles to $\mathcal{A L C}(\mathbf{R})$, the extension of $\mathcal{A L C}$ by an admissible concrete domain $\mathbf{R}$ based on real arithmetics, renders the subsumption problem undecidable. The proof of this result uses a reduction from the Post Correspondence Problem (PCP). It was shown in [31] that this proof can be adapted to the case where transitive closure of roles is replaced by GCIs, and it actually works for considerably weaker concrete domain, such as the rational numbers $\mathbb{Q}$ or the natural numbers $\mathbb{N}$ with a unary predicate $=_{0}$ for equality with zero, a binary equality predicate $=$, and a unary predicate $+_{1}$ for incrementation. In [7] it is shown, by a reduction from the halting problem of two-register machines, that undecidability even holds without binary equality. In the present paper, we will improve on this result by showing that, even if $=_{0}$ is removed as well, undecidability still holds, and that the same is true if we replace $+_{1}$ with + .

To regain decidability, one can either impose syntactic restriction on how the DL can interact with the concrete domain $[22,36]$. The main idea is here to disallow paths (such as child age in our example), which has the effect that concrete domain predicates cannot compare properties (such as the age) of different individuals. The other option is to impose stronger restrictions than admissibility on the concrete domain. After first positive results for specific concrete domains (e.g., a concrete domain over the rational numbers with order and equality $[30,32])$, the notion of $\omega$-admissible concrete domains was introduced in [33], and it was shown (by designing a tableau-based decision procedure) that integrating such a concrete domain into $\mathcal{A L C}$ leaves reasoning decidable also in the presence of GCIs. In the present paper, we generalize the notion of $\omega$ admissibility and the decidability result from concrete domains with only binary predicates as in [33] to concrete domains with predicates of arbitrary arity. But the main contribution of this paper is to show that there is a close relationship between $\omega$-admissibility and well-known notions from model theory. In particular, we show that finitely bounded, homogeneous structures yield $\omega$-admissible concrete domains. This allows us to locate new $\omega$-admissible concrete domains using existing results from model theory. Due to space constraints, we cannot prove all our results in detail here. Complete proofs and more examples of $\omega$ admissible concrete domains can be found in [8].

\section{Preliminaries}

We write $[n]$ for the set $\{1, \ldots, n\}$. Given a set $A$, the diagonal on $A$ is defined as the binary relation $\triangle_{A}:=\{(a, a) \mid a \in A\}$. The kernel of a mapping $f: A \rightarrow B$, denoted by ker $f$, is the equivalence relation $\left\{\left(a, a^{\prime}\right) \in A \times A \mid f(a)=f\left(a^{\prime}\right)\right\}$.

From a mathematical point of view, concrete domains are relational structures. A (relational) signature $\tau$ is a set of predicate symbols, each with an associated natural number called its arity. A relational $\tau$-structure $\mathbf{A}$ consists of a set $A$ (the domain) together with relations $R^{\mathbf{A}} \subseteq A^{k}$ for each $k$-ary symbol $R \in \tau$. We often describe structures by listing their domain and relations, e.g., 
we write $\mathbf{Q}=(\mathbb{Q} ;<)$ for the relational structure whose domain is the set of rational numbers $\mathbb{Q}$, and which has the usual smaller relation $<$ on $\mathbb{Q}$ as its only relation. ${ }^{2}$ An expansion of the $\tau$-structure $\mathbf{A}$ is a $\sigma$-structure $\mathbf{B}$ with $A=B$, $\tau \subseteq \sigma$, and $R^{\mathbf{B}}=R^{\mathbf{A}}$ for each relation symbol $R \in \tau$. Conversely, we call $\mathbf{A}$ a reduct of $\mathbf{B}$.

One possibility to obtain an expansion of a $\tau$-structure is to use formulas of first-order logic (FO) over the signature $\tau$ to define new predicates, where a formula with $k$ free variables defines a $k$-ary predicate in the obvious way. We assume that equality $=$ as well as the symbol false for falsity is always available. Thus, atomic formulas are of the form false, $x_{i}=x_{j}$, and $R\left(x_{1}, \ldots, x_{k}\right)$ for some $k$-ary $R \in \tau$ and variables $x_{1}, \ldots, x_{k}$. The FO theory of a structure is the set of all FO sentences that are true in the structure. In addition to full FO, we also use standard fragments of FO such as the existential positive $\left(\exists^{+}\right)$, the quantifier-free (qf), and the primitive positive (pp) fragment. The existential positive fragment consists of formulas built using conjunction, disjunction, and existential quantification only. The quantifier-free fragment consists of Boolean combinations of atomic formulas, and the primitive positive fragment of existentially quantified conjunctions of atomic formulas. Let $\Sigma$ be a set of FO formulas and $\mathbf{D}$ a structure. We say that a relation over $D$ has a $\Sigma$ definition in $\mathbf{D}$ if it is of the form $\left\{t \in D^{k} \mid \mathbf{D} \models \phi(t)\right\}$ for some $\phi \in \Sigma$. We refer to this relation by $\phi^{\mathbf{D}}$. For example, the formula $y<x \vee x=y$ is an existential positive formula and, interpreted in the structure $\mathbf{Q}$, it clearly defines the binary relation $\geq$ on $\mathbb{Q}$. This shows that $\geq$ is $\exists^{+}$definable in $\mathbf{Q}$. An example of a pp formula is the formula $\exists y \cdot x=y$, which defines the unary relation interpreted as the whole domain $\mathbb{Q}$.

A homomorphism $h: \mathbf{A} \rightarrow \mathbf{B}$ for $\tau$-structures $\mathbf{A}, \mathbf{B}$ is a mapping $h: A \rightarrow$ $B$ that preserves each relation of $\mathbf{A}$, i.e., $\left(a_{1}, \ldots, a_{k}\right) \in R^{\mathbf{A}}$ for some $k$-ary relation symbol $R \in \tau$ implies $\left(h\left(a_{1}\right), \ldots, h\left(a_{k}\right)\right) \in R^{\mathbf{B}}$. We write $\mathbf{A} \rightarrow \mathbf{B}$ if $\mathbf{A}$ homomorphically maps to $\mathbf{B}$ and $\mathbf{A} \nrightarrow \mathbf{B}$ otherwise. We say that $\mathbf{A}$ and $\mathbf{B}$ are homomorphically equivalent if $\mathbf{A} \rightarrow \mathbf{B}$ and $\mathbf{B} \rightarrow \mathbf{A}$. An endomorphism is a homomorphism from a structure to itself. By an embedding we mean an injective homomorphism that additionally satisfies the only if direction in the definition of a homomorphism, i.e., it also preserves the complements of relations. We write $\mathbf{A} \hookrightarrow \mathbf{B}$ if $\mathbf{A}$ embeds into $\mathbf{B}$. A substructure of $\mathbf{A}$ is a structure $\mathbf{B}$ over $B \subseteq A$ such that the natural inclusion map $i: A \rightarrow B$ is an embedding. We call $\mathbf{A}$ an extension of $\mathbf{B}$. An isomorphism is a surjective embedding. We say that two structures $\mathbf{A}$ and $\mathbf{B}$ are isomorphic and write $\mathbf{A} \cong \mathbf{B}$ if there exists an isomorphism from $\mathbf{A}$ to $\mathbf{B}$. An automorphism is an isomorphism from a structure into itself.

The definition of admissibility of a concrete domain in [4] requires that the constraint satisfaction problem for this structure is decidable. Let $\mathbf{D}$ be a structure with a finite relational signature $\tau$. The constraint satisfaction problem of $\mathbf{D}$, short $\operatorname{CSP}(\mathbf{D})$, is the following decision problem:

\footnotetext{
$\overline{{ }^{2} \text { By a slight }}$ abuse of notation, we use $<$ instead of $<^{\mathbf{Q}}$ to denote also the interpretation of the predicate symbol $<$ in $\mathbf{Q}$.
} 
INPUT: A finite $\tau$-structure A.

QUESTION: Does A homomorphically map to D?

Formally, $\operatorname{CSP}(\mathbf{D})$ is the class of all finite $\tau$-structures that homomorphically map to $\mathbf{D}$. We call $\mathbf{D}$ the template of $\operatorname{CSP}(\mathbf{D})$. A solution for an instance $\mathbf{A}$ of the CSP is a homomorphism $h: \mathbf{A} \rightarrow \mathbf{D}$.

It is easy to see that deciding whether a CSP instance admits a solution amounts to evaluating a pp sentences in the template and vice versa [10]. For example, verifying whether the structure $\mathbf{A}=\left(\left\{a_{1}, a_{2}, a_{3}\right\} ;<^{\mathbf{A}}\right)$ with $<^{\mathbf{A}}:=$ $\left\{\left(a_{1}, a_{2}\right),\left(a_{2}, a_{3}\right),\left(a_{3}, a_{1}\right)\right\}$ homomorphically maps into $\mathbf{Q}$ is the same as checking whether the pp sentence $\exists x_{1} . \exists x_{2} . \exists x_{3} .\left(x_{1}<x_{2} \wedge x_{2}<x_{3} \wedge x_{3}<x_{1}\right)$ is true in $\mathbf{Q}$.

The CSP for $\mathbf{Q}$ is in $\mathbf{P}$ since a structure $\mathbf{A}=\left(A,<^{\mathbf{A}}\right)$ can homomorphically be mapped into $\mathbb{Q}$ iff it does not contain a $<$-cycle, i.e., there are is no $n \geq 1$ and elements $a_{0}, \ldots, a_{n-1} \in A$ such that $a_{0}<^{\mathbf{A}} \ldots<^{\mathbf{A}} a_{n-1}<{ }^{\mathbf{A}} a_{0}$. Testing whether such a cycle exists can be done in logarithmic space since it requires solving the reachability problem in a directed graph (digraph). In the example above, we obviously have a cycle, and thus this instance of $\operatorname{CSP}(\mathbf{Q})$ has no solution.

The definition of admissibility in [4] actually also requires that the predicates are closed under negation and that there is a predicate for the whole domain. We have already seen that the negation $\geq$ of $<$ is $\exists^{+}$definable in $\mathbf{Q}$ and that the predicate for the whole domain is pp definable. The negation of this predicate has the pp definition $x<x$. The following lemma implies that the expansion of $\mathbf{Q}$ by these predicates still has a decidable CSP. ${ }^{3}$

Lemma 1 ([10]). Let $\mathbf{C , D}$ be structures over the same domain with finite signatures. If the relations of $\mathbf{C}$ have a pp definition in $\mathbf{D}$, then $\operatorname{CSP}(\mathbf{C}) \leq_{\text {PTIME }}$ $\operatorname{CSP}(\mathbf{D})$; if they have an $\exists^{+}$definition in $\mathbf{D}$, then $\operatorname{CSP}(\mathbf{C}) \leq_{\text {NPTIME }} \operatorname{CSP}(\mathbf{D})$.

\section{DLs with Concrete Domains}

As in [4] and [33], we extend the well-known DL $\mathcal{A L C}$ with concrete domains. We assume that the reader is familiar with the syntax and semantics of $\mathcal{A L C}$ (as, e.g., defined in [7]), and thus only show how both need to be extended to accommodate a concrete domain $\mathbf{D}$. In the general definition, we allow reference to $\Sigma$ definable predicates for a fragment $\Sigma$ of FO rather than just the elements of $\tau$. For technical reasons, we must, however, restrict the arities of definable predicates by a fixed upper bound $d$. Given a $\tau$-structure $\mathbf{D}$ with finite relational signature $\tau$, a set $\Sigma$ of FO formulas over the signature $\tau$, and a bound $d \geq 1$ on the arity of the $\Sigma$-definable predicates, we obtain a $\mathrm{DL} \mathcal{A L C}_{\Sigma}^{d}(\mathbf{D})$, which extends $\mathcal{A L C}$ as follows.

From a syntactic point of view, we assume that the set of role names $N_{R}$ contains a set of functional roles $\mathrm{N}_{\mathrm{fR}} \subseteq \mathrm{N}_{\mathrm{R}}$, and that in addition we have a set of

\footnotetext{
${ }^{3}$ The lemma actually only yields an NP decision procedure for this CSP, but it is easy to see that the above polynomial-time cycle-checking algorithm can be adapted such that it also works for the expanded structure.
} 
feature names $N_{F}$, which provide the connection between the abstract and the concrete domain. A path is of the form $r f$ or $f$ where $r \in \mathrm{N}_{\mathrm{R}}$ and $f \in \mathrm{N}_{\mathrm{F}}$. In our example in the introduction, age is a feature name and childage is a path. The DL $\mathcal{A} \mathcal{L C}_{\Sigma}^{d}(\mathbf{D})$ extends $\mathcal{A L C}$ with two new concept constructors:

$$
\exists p_{1}, \ldots, p_{k} \cdot\left[\phi\left(x_{1}, \ldots, x_{k}\right)\right] \text { and } \forall p_{1}, \ldots, p_{k} \cdot\left[\phi\left(x_{1}, \ldots, x_{k}\right)\right] \text {, }
$$

where $k \leq d, p_{1}, \ldots, p_{k}$ are paths, and $\phi\left(x_{1}, \ldots, x_{k}\right)$ is a formula in $\Sigma$ with $k$ free variables, defining a $k$-ary predicate on $D$. As usual, a TBox is defined to be a finite set of GCIs $C \sqsubseteq D$, where $C, D$ are $\mathcal{A L C} \mathcal{C}_{\Sigma}^{d}(\mathbf{D})$ concept descriptions.

Regarding the semantics, we consider interpretations $\mathcal{I}=\left(\Delta^{\mathcal{I}},{ }^{\mathcal{I}}\right)$, consisting of a non-empty set $\Delta^{\mathcal{I}}$ and an interpretation function ${ }^{\mathcal{I}}$, which interprets concept names $A$ as subsets $A^{\mathcal{I}}$ of $\Delta^{\mathcal{I}}$ and role names $r$ as binary relations $r^{\mathcal{I}}$ on $\Delta^{\mathcal{I}}$, with the restriction that $r^{\mathcal{I}}$ is functional for $r \in \mathrm{N}_{\mathrm{fR}}$, i.e., $(d, e) \in r^{\mathcal{I}}$ and $\left(d, e^{\prime}\right) \in r^{\mathcal{I}}$ imply $e=e^{\prime}$. In addition, features $f \in \mathrm{N}_{\mathrm{F}}$ are interpreted as functional binary relations $f^{\mathcal{I}} \subseteq \Delta^{\mathcal{I}} \times D$. We extend the interpretation function to paths of the form $p=r f$ by setting

$$
p^{\mathcal{I}}=\left\{\left(d, d^{\prime}\right) \mid \text { there is } d^{\prime \prime} \in \Delta^{\mathcal{I}} \text { such that }\left(d, d^{\prime \prime}\right) \in r^{\mathcal{I}} \text { and }\left(d^{\prime \prime}, d^{\prime}\right) \in f^{\mathcal{I}}\right\} .
$$

For the concept constructors of $\mathcal{A L C}$, the extension of the interpretation function to complex concepts is defined in the usual way. The new concrete domain constructors are interpreted as follows:

$$
\begin{array}{r}
\left(\exists p_{1}, \ldots, p_{k} \cdot\left[\phi\left(x_{1}, \ldots, x_{k}\right)\right]\right)^{\mathcal{I}}=\left\{d \in \Delta^{\mathcal{I}} \mid \text { there exist } d_{1}, \ldots, d_{k} \in D\right. \text { such that } \\
\left.\left(d, d_{i}\right) \in p_{i}^{\mathcal{I}} \text { for all } i \in[k] \text { and } \mathbf{D} \models \phi\left(d_{1}, \ldots, d_{k}\right)\right\}, \\
\left(\forall p_{1}, \ldots, p_{k} \cdot\left[\phi\left(x_{1}, \ldots, x_{k}\right)\right]\right)^{\mathcal{I}}=\left\{d \in \Delta^{\mathcal{I}} \mid \text { for all } d_{1}, \ldots, d_{k} \in D\right. \text { we have that } \\
\left.\left(d, d_{i}\right) \in p_{i}^{\mathcal{I}} \text { for all } i \in[k] \text { implies } \mathbf{D} \models \phi\left(d_{1}, \ldots, d_{k}\right)\right\} .
\end{array}
$$

As usual, an interpretation $\mathcal{I}$ is a model a TBox $\mathcal{T}$ if it satisfies all the GCIs in $\mathcal{T}$, where $\mathcal{I}$ satisfies the GCI $C \sqsubseteq D$ if $C^{\mathcal{I}} \subseteq D^{\mathcal{I}}$ holds. The $\mathcal{A L C}_{\Sigma}^{d}(\mathbf{D})$ concept description $C$ is satisfiable w.r.t. $\mathcal{T}$ if there is a model $\mathcal{I}$ of $\mathcal{T}$ such that $C^{\mathcal{I}} \neq \emptyset$. Since all Boolean operators are available in $\mathcal{A L C}_{\Sigma}^{d}(\mathbf{D})$, the subsumption problem mentioned in the introduction and the satisfiability problem are inter-reducible in polynomial time [7].

As a convention, we write $\mathcal{A L C}(\mathbf{D})$ instead of $\mathcal{A L C}_{\Sigma}^{d}(\mathbf{D})$ if $d$ is the maximal arity of the predicates in $\tau$ and $\Sigma$ consists of all atomic $\tau$-formulas not using the equality predicate.

\subsection{Undecidable DLs with Concrete Domains}

We show by a reduction from the halting problem of two-register machines that concept satisfiability in $\mathcal{A L C}(\mathbf{D})$ is undecidable if $\mathbf{D}$ is a structure with domain $\mathbb{Q}, \mathbb{Z}$, or $\mathbb{N}$ whose only predicate is the binary predicate $+_{1}$, which is interpreted as incrementation (i.e., it consists of the tuples $(m, m+1)$ for numbers $m$ in the respective domain).

Our proof is an adaptation of the undecidability proof in [7] to the case where no zero test $=_{0}$ is available. ${ }^{4} \mathrm{~A}$ two-register machine $(2 \mathrm{RM})$ is a pair $(Q, P)$

\footnotetext{
${ }^{4}$ A similar trick for zero test elimination is used in the proof of Proposition 1 in [16].
} 
with states $Q=\left\{q_{0}, \ldots, q_{\ell}\right\}$ and a sequence of instructions $P=I_{0}, \ldots, I_{\ell-1}$. By definition, $q_{0}$ is the initial state and $q_{\ell}$ the halting state. In state $q_{i}(i<$ $\ell$ ) the instruction $I_{i}$ must be applied. Instructions come in two varieties. An incrementation instruction is of the form $I=+(p, q)$ where $p \in\{1,2\}$ is the register number and $q$ is a state. This instruction increments (the content of) register $p$ and then goes to state $q$. A decrementation instruction is of the form $I=-\left(p, q, q^{\prime}\right)$ where $p \in\{1,2\}$ and $q, q^{\prime}$ are states. This instruction decrements register $p$ and goes to state $q$ if the content of register $p$ is not zero; otherwise, it leaves register $p$ as it is and goes to state $q^{\prime}$. It is well-known that the problem of deciding whether a given 2RM halts on input $(0,0)$ is undecidable [35].

Proposition 1. If $\mathbf{D}$ is $\left(\mathbb{Q},+_{1}\right),\left(\mathbb{Z},+_{1}\right)$, or $\left(\mathbb{N},+_{1}\right)$, then concept satisfiability in $\mathcal{A L C}(\mathbf{D})$ w.r.t. TBoxes is undecidable.

Proof. Let $(Q, P)$ be an arbitrary 2RM. We define a concept $C$ and a TBox $\mathcal{T}$ in such a way that every model of $\mathcal{T}$ in which $C$ is non-empty represents the computation of $(Q, P)$ on the input $(0,0)$. For every state $q_{i}$ we introduce a concept name $Q_{i}$. We also introduce two concept names $Z_{1}, Z_{2}$ to indicate a positive zero test for the first and second register, respectively. In addition, we introduce a functional role $g \in \mathrm{N}_{\mathrm{fR}}$ representing the transitions between configurations of the 2RM. For $p \in\{1,2\}$, we have features $r_{p} \in \mathrm{N}_{\mathrm{F}}$ representing the content of register $p$. However, since our concrete domain does not have the predicate $=_{0}$, we cannot enforce that, in our representation of the initial configuration, $r_{1}$ and $r_{2}$ have value zero. What we can ensure, though, is that their value is the same number, which we can store in a concrete feature $z \in N_{\mathrm{F}}$. The idea is now that register $p$ of the machine actually contain the value of $r_{p}$ offset with the value of $z$. We also need auxiliary concrete features $s_{1}, s_{2}, s \in \mathrm{N}_{\mathrm{F}}$, which respectively refer to the successor values of $r_{1}, r_{2}, z$. They are needed to express equality using $+_{1}$.

The following GCI ensures that the elements of $C$ represent the initial configuration together with appropriate values for the auxiliary features:

$$
C \sqsubseteq Q_{0} \sqcap \exists r_{1}, s_{1} \cdot\left[+_{1}\right] \sqcap \exists r_{2}, s_{2} \cdot\left[+_{1}\right] \sqcap \exists z, s_{1} \cdot\left[+_{1}\right] \sqcap \exists z, s_{2} \cdot\left[+_{1}\right] \sqcap \exists z, s \cdot\left[+_{1}\right] .
$$

Next, the GCI $\top \sqsubseteq \exists g z, s .\left[+_{1}\right] \sqcap \exists g z, g s .\left[+_{1}\right]$ guarantees that the value $z$ of an individual carries over to its $g$-successor. We denote the second value in $\{1,2\}$ beside $p$ by $\widehat{p}$, i.e., $\widehat{p}=3-p$. To enforce that the incrementation instructions are executed correctly, for every instruction $I_{i}=+\left(p, q_{j}\right)$, we include in $\mathcal{T}$ the GCI

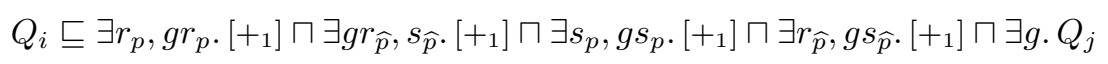

The GCIs $Z_{p} \sqsubseteq \exists z, s_{p} \cdot\left[+_{1}\right], \exists z, s_{p} \cdot\left[+_{1}\right] \sqsubseteq Z_{p}$ ensure that $Z_{p}$ represents a positive zero test for register $p$. Note that, for individuals for which values for $s, z, s_{p}, r_{p}$ are defined, the negation of $Z_{p}$ is semantically equivalent to a negative zero test for register $p$. To enforce that decrementation is executed correctly, for every instruction $I_{i}=-\left(p, q_{j}, q_{k}\right)$, we include in $\mathcal{T}$ the GCIs

$$
\begin{aligned}
& Q_{i} \sqcap Z_{p} \sqsubseteq \exists g r_{p}, s_{p} \cdot\left[+_{1}\right] \sqcap \exists g r_{\widehat{p}}, s_{\widehat{p}} \cdot\left[+_{1}\right] \sqcap \exists r_{p}, g s_{p} \cdot\left[+_{1}\right] \sqcap \exists r_{\widehat{p}}, g s_{\widehat{p}} \cdot\left[+_{1}\right] \sqcap \exists g \cdot Q_{k}, \\
& Q_{i} \sqcap \neg Z_{p} \sqsubseteq \exists g r_{p}, r_{p} \cdot\left[+_{1}\right] \sqcap \exists g r_{\widehat{p}}, s_{\widehat{p}} \cdot\left[+_{1}\right] \sqcap \exists g s_{p}, s_{p} \cdot\left[+_{1}\right] \sqcap \exists r_{\widehat{p}}, g s_{\widehat{p}} \cdot\left[+_{1}\right] \sqcap \exists g \cdot Q_{j} .
\end{aligned}
$$


Finally, we include the GCI $Q_{\ell} \sqsubseteq \perp$, which states that the halting state is never reached. It is now easy to see that the computation of $(Q, P)$ on $(0,0)$ does not reach the halting state iff $C$ is satisfiable w.r.t. $\mathcal{T}$.

Note that, even though our proof of Proposition 1 uses a functional role $g$ to represent the transitions between configurations of a given two-register machine, the reduction also works if $g$ is assumed to be an arbitrary role. One simply must use additional universal quantification to ensure that all the $g$-successors of an individual behave the same (i.e., for every existential quantification in the current proof we add the corresponding universal quantification).

It turns out that undecidability also holds if we use the ternary predicate + rather than the binary predicate $+_{1}$. Intuitively, with + we can easily test for 0 since $m$ is 0 iff $m+m=m$. Instead of incrementation by 1 , we can then use addition of a fixed non-zero number (see [8] for a detailed proof).

Proposition 2. If $\mathbf{D}$ is $(\mathbb{Q},+),(\mathbb{Z},+)$, or $(\mathbb{N},+)$, then concept satisfiability in $\mathcal{A L C}(\mathbf{D})$ w.r.t. TBoxes is undecidable.

\section{$3.2 \omega$-admissible Concrete Domains}

To regain decidability in the presence of GCIs and concrete domains, the notion of $\omega$-admissible concrete domains was introduced in [33]. We generalize this notion and the decidability result from concrete domains with only binary predicates as in [33] to concrete domains with predicates of arbitrary arity.

We say that a countable structure $\mathbf{D}$ has homomorphism compactness if, for every countable structure $\mathbf{B}$, it holds that $\mathbf{B} \rightarrow \mathbf{D}$ iff $\mathbf{A} \rightarrow \mathbf{D}$ for every finite structure $\mathbf{A}$ with $\mathbf{A} \hookrightarrow \mathbf{B}$. A relational $\tau$-structure $\mathbf{D}$ satisfies

(JE) if, for some $d \geq 2, \bigcup\left\{R^{\mathbf{D}} \mid R \in \tau, R^{\mathbf{D}} \subseteq D^{k}\right\}=D^{k}$ if $k \leq d$ and $\emptyset$ else; (PD) if $R^{\mathbf{D}} \cap R^{\prime \mathbf{D}}=\emptyset$ for all pairwise distinct $R, R^{\prime} \in \tau$; (JD) if $\bigcup\left\{R^{\mathbf{D}} \mid R \in \tau, R^{\mathbf{D}} \subseteq \triangle_{D}\right\}=\triangle_{D}$.

Here JE stands for "jointly exhaustive," PD for "pairwise disjoint," and JD for "jointly diagonal." Note that JD was not considered in [33]. We include it here since it makes the comparison with known notions from model theory easier. In addition, all the $\omega$-admissible concrete domains considered in [33] satisfy JD.

A relational $\tau$-structure $\mathbf{D}$ is a patchwork if it is JDJEPD, and for all finite JEPD $\tau$-structures $\mathbf{A}, \mathbf{B}_{1}, \mathbf{B}_{2}$ with $e_{1}: \mathbf{A} \hookrightarrow \mathbf{B}_{1}, e_{2}: \mathbf{A} \hookrightarrow \mathbf{B}_{2}, \mathbf{B}_{1} \rightarrow \mathbf{D}$ and $\mathbf{B}_{2} \rightarrow \mathbf{D}$, there exist $f_{1}: \mathbf{B}_{1} \rightarrow \mathbf{D}$ and $f_{2}: \mathbf{B}_{2} \rightarrow \mathbf{D}$ with $f_{1} \circ e_{1}=f_{2} \circ e_{2}$.

Definition 1. The relational structure $\mathbf{D}$ is $\omega$-admissible if $\operatorname{CSP}(\mathbf{D})$ is decidable, $\mathbf{D}$ has homomorphism compactness, and $\mathbf{D}$ is a patchwork.

The idea is now that one can use disjunctions of atomic formulas of the same arity within concrete domain restrictions. We refer to the set of all FO $\tau$-formulas of the form $R_{1}\left(x_{1}, \ldots, x_{k}\right) \vee \cdots \vee R_{m}\left(x_{1}, \ldots, x_{k}\right)$ for $R_{1}, \ldots, R_{m} k$-ary predicates in $\tau$ by $\vee^{+}$. The following theorem is proved in [8] by extending the tableau-based decision procedure given in [33] to our more general definition of $\omega$-admissibility. Note that the proof of correctness of this procedure does not depend on JD. 
Theorem 1. Let $\mathbf{D}$ be an $\omega$-admissible $\tau$-structure with at most d-ary relations for some $d \geq 2$. Then concept satisfiability in $\mathcal{A L C}_{\mathrm{V}^{+}}(\mathbf{D})$ w.r.t. TBoxes is decidable.

The main motivation for the definition of $\omega$-admissible concrete domains in [33] was that they can capture qualitative calculi of time and space. In particular, it was shown in [33] that Allen's interval logic [1] as well as the region connection calculus RCC8 [37] can be represented as $\omega$-admissible concrete domains. To the best of our knowledge, no other $\omega$-admissible concrete domains have been exhibited in the literature since then.

\section{A Model-Theoretic Approach Towards $\omega$-admissibility}

In this section, we introduce several model-theoretic properties of relational structures and show their connection to $\omega$-admissibility. This allows us to formulate sufficient conditions for $\omega$-admissibility using well-know notions from model theory, and thus to employ existing model-theoretic results to find new $\omega$-admissible concrete domains.

$\boldsymbol{\omega}$-categoricity. We start with introducing $\omega$-categoricity since it gives us homomorphism compactness "for free." A structure is $\omega$-categorical if its first-order theory has exactly one countable model up to isomorphism. For example, it is well-known that $\mathbf{Q}$ is, up to isomorphism, the only countable dense linear order without lower or upper bound. This result, which clearly implies that $\mathbf{Q}$ is $\omega$-categorical, is due to Cantor.

For every structure $\mathbf{A}$, the set of all its automorphisms forms a permutation group, which we denote by $\operatorname{Aut}(\mathbf{A})$ (see Theorem 1.2.1 in [25]). Every relation with an FO definition in $\mathbf{A}$ is easily seen to be preserved by $\operatorname{Aut}(\mathbf{A})$. For $\omega$ categorical structures, the other direction holds as well.

Theorem 2 (Engeler, Ryll-Nardzewski, Svenonius [25]). For a countably infinite structure $\mathbf{A}$ with a countable signature, the following are equivalent:

1. A is $\omega$-categorical.

2. For every $k \geq 1$, only finitely many $k$-ary relations are $F O$ definable in $\mathbf{A}$.

3. Every relation over $A$ preserved by $A$ ut $(\mathbf{A})$ is FO definable in $\mathbf{A}$.

The following corollary to this theorem establishes the first link between model theory and $\omega$-admissibility.

Corollary 1 (Lemma 3.1.5 in [10]). Every $\omega$-categorical structure has homomorphism compactness.

In order to obtain JDJEPD, we replace the original relations of a given $\omega$-categorical $\tau$-structure $\mathbf{A}$ with appropriate first-order definable ones, using the results of Theorem 2. The orbit of a tuple $\left(a_{1}, \ldots, a_{k}\right) \in A^{k}$ under the natural action of $\operatorname{Aut}(\mathbf{A})$ on $A^{k}$ is the set $\left\{\left(g\left(a_{1}\right), \ldots, g\left(a_{n}\right)\right) \mid g \in \operatorname{Aut}(\mathbf{A})\right\}$. By Theorem 2 , the set of all at most $k$-ary relations $\mathrm{FO}$ definable in $\mathbf{A}$ is finite 
for every $k \in \mathbb{N}$. Since every such set is closed under intersections, it contains finitely many minimal non-empty relations. Since every relation over $A$ that is preserved by all automorphisms of $\mathbf{A}$ is $\mathrm{FO}$ definable in $\mathbf{A}$, these minimal elements are precisely the orbits of tuples over $A$ under the natural action of $\operatorname{Aut}(\mathbf{A})$.

Definition 2. For a given arity bound $d \geq 2$, the $d$-reduct of the $\omega$-categorical $\tau$ structure $\mathbf{A}$, denoted by $\mathbf{A}^{\leqslant d}$, is the relational structure over $A$ whose relations are all orbits of at most d-ary tuples over $A$ under $A$ ut $(\mathbf{A})$. We denote the signature of $\mathbf{A} \leqslant d$ by $\tau^{\leqslant d}$.

It is easy to see that $\mathbf{A} \leqslant d$ is JDJEPD, and that every at most $d$-ary relation over $A$ FO definable in $\mathbf{A}$ can be obtained as a disjunction of atomic formulas built using the symbols in $\tau^{\leqslant d}$. As an example, consider the $\omega$-categorical structure $\mathbf{Q}$. The orbits of $k$-tuples of elements of $\mathbb{Q}$ can be defined by quantifierfree formulas that are conjunctions of atoms of the form $x_{i}=x_{j}$ or $x_{i}<x_{j}$. For example, the orbit of the tuple $(2,3,2,5)$ consists of all tuples $\left(q_{1}, q_{2}, q_{3}, q_{4}\right) \in \mathbb{Q}^{4}$ that satisfy the formula $x_{1}<x_{2} \wedge x_{1}=x_{3} \wedge x_{2}<x_{4}$ if $x_{i}$ is replaced by $q_{i}$ for $i=1, \ldots, 4$. The FO definable $k$-ary relations in $\mathbf{Q}$ are obtained as unions of these orbits, where the defining formula is then the disjunction of the formulas defining the respective orbits. Since these formulas are quantifier-free, this also shows that $\mathbf{Q}$ admits quantifier elimination. Recall that a $\tau$-structure admits quantifier elimination if for every $\mathrm{FO} \tau$-formula there exists a quantifier-free (qf) $\tau$-formula that defines the same relation over this structure.

Homogeneity. To obtain the patchwork property, we restrict the attention to homogeneous structures. A structure $\mathbf{A}$ is homogeneous if every isomorphism between finite substructures of $\mathbf{A}$ extends to an automorphism of $\mathbf{A}$.

Theorem 3 ([25]). A countable relational structure with a finite signature is homogeneous iff it is $\omega$-categorical and admits quantifier elimination.

Since $\mathbf{Q}$ is $\omega$-categorical and admits quantifier elimination, it is thus homogeneous. This can, however, also easily be shown directly without using the theorem. In fact, given finite substructures $\mathbf{B}$ and $\mathbf{C}$ of $\mathbf{Q}$ and an isomorphism between them, we know that $B$ consists of finitely many elements $p_{1}, \ldots, p_{n}$ and $C$ of the same number of elements $q_{1}, \ldots, q_{n}$ such that $p_{1}<\ldots<p_{n}$, $q_{1}<\ldots<q_{n}$, and the isomorphism maps $p_{i}$ to $q_{i}$ (for $i=1, \ldots, n$ ). It is now easy to see that $<$ is also a dense linear order without lower or upper bound on the sets $\left\{p \mid p<p_{1}\right\}$ and $\left\{q \mid q<q_{1}\right\}$, and thus there is an order isomorphism between these sets. The same is true for the pairs of sets $\left\{p \mid p_{i}<p<p_{i+1}\right\}$ and $\left\{q \mid q_{i}<q<q_{i+1}\right\}$, and for the pair $\left\{p \mid p_{n}<p\right\}$ and $\left\{q \mid q<q_{n}\right\}$. Using the isomorphisms between these pairs, we can clearly put together an isomorphisms from $\mathbf{Q}$ to $\mathbf{Q}$ that extends the original isomorphism from $\mathbf{B}$ to $\mathbf{C}$.

Countable homogeneous structures can be obtained as Fraïssé limits of amalgamation classes. A class $\mathcal{K}$ of relational $\tau$-structures has the amalgamation property (AP) if, for every $\mathbf{A}, \mathbf{B}_{1}, \mathbf{B}_{2} \in \mathcal{K}$ with $e_{1}: \mathbf{A} \hookrightarrow \mathbf{B}_{1}$ and $e_{2}: \mathbf{A} \hookrightarrow \mathbf{B}_{2}$ there 
exists $\mathbf{C} \in \mathcal{K}$ with $f_{1}: \mathbf{B}_{1} \hookrightarrow \mathbf{C}$ and $f_{2}: \mathbf{B}_{2} \hookrightarrow \mathbf{C}$ such that $f_{1} \circ e_{1}=f_{2} \circ e_{2}$. A class $\mathcal{K}$ of finite relational structures with a countable signature $\tau$ is called an amalgamation class if it has AP, is closed under applying isomorphisms and taking substructures, and contains only countably many structures up to isomorphism. We denote by $\operatorname{Age}(\mathbf{A})$ the class of all finite structures that embed into the structure $\mathbf{A}$.

Theorem 4 (Fraïssé [25]). Let $\mathcal{K}$ be an amalgamation class of $\tau$-structures. Then there exists a homogeneous countable $\tau$-structure $\mathbf{A}$ with Age $(\mathbf{A})=\mathcal{K}$. The structure $\mathbf{A}$ is unique up to isomorphism and referred to as the Fraïssé limit of $\mathcal{K}$. Conversely, Age (A) for a countable homogeneous structure $\mathbf{A}$ with a countable signature is an amalgamation class.

For our running example $\mathbf{Q}=(\mathbb{Q},<)$, we have that $\operatorname{Age}(\mathbf{Q})$ consists of all finite linear orders, and thus by Fraïssé's theorem this class of structures is an amalgamation class. In addition, $\mathbf{Q}$ is the Fraïssé limit of this class. Proposition 3 below shows that there is a close connection between AP and the patchwork property. Its proof uses the following lemma, whose proof can be found in [8].

Lemma 2. Let $\mathbf{A}, \mathbf{B}$ be two JEPD $\tau$-structures, such that $\mathbf{B}$ is $J D$, and $f: \mathbf{A} \rightarrow$ $\mathbf{B}$ a homomorphism. Then $f$ preserves the complements of all relations of $\mathbf{A}$ and ker $f=\bigcup\left\{R^{\mathbf{A}} \mid R \in \tau\right.$ and $\left.R^{\mathbf{B}} \subseteq \triangle_{B}\right\}$.

Proposition 3. Let $\mathbf{D}$ be a JDJEPD $\tau$-structure. Then $\mathbf{D}$ is a patchwork iff Age (D) has AP.

Proof. For simplification purposes, every statement indexed by $i$ is suppose to hold for both $i \in\{1,2\}$. First, suppose that Age (D) has AP. Let $\mathbf{A}, \mathbf{B}_{1}, \mathbf{B}_{2}$ be finite JEPD $\tau$-structures with $e_{i}: \mathbf{A} \hookrightarrow \mathbf{B}_{i}$ and $h_{i}: \mathbf{B}_{i} \rightarrow \mathbf{D}$. We must show that there exist $f_{i}: \mathbf{B}_{i} \rightarrow \mathbf{D}$ with $f_{1} \circ e_{1}=f_{2} \circ e_{2}$. Let $\widehat{\mathbf{A}}_{1}$ and $\widehat{\mathbf{A}}_{2}$ be the substructures of $\mathbf{D}$ on $\left(h_{1} \circ e_{1}\right)(A)$ and $\left(h_{2} \circ e_{2}\right)(A)$, respectively. Clearly both $\widehat{\mathbf{A}}_{1}$ and $\widehat{\mathbf{A}}_{2}$ are JDJEPD, because they are substructures of $\mathbf{D}$. Due to Lemma 2 , we have $\widehat{\mathbf{A}}_{1} \cong \widehat{\mathbf{A}}_{2}$, because both $h_{1} \circ e_{1}$ and $h_{2} \circ e_{2}$ preserve the complements of all relations of $\mathbf{A}$ and $\operatorname{ker} h_{1} \circ e_{1}=\bigcup\left\{R^{\mathbf{A}} \mid R \in \tau\right.$ and $\left.R^{\mathbf{D}} \subseteq \triangle_{D}\right\}=\operatorname{ker} h_{2} \circ e_{2}$.

However, what we want is an isomorphism that commutes with $h_{1} \circ e_{1}$ and $h_{2} \circ e_{2}$. Consider the map $g: \widehat{A}_{1} \rightarrow \widehat{A}_{2}$ given by $g\left(\left(h_{1} \circ e_{1}\right)(a)\right):=\left(h_{2} \circ e_{2}\right)(a)$. It is well defined, because $\operatorname{ker} h_{1} \circ e_{1}=\operatorname{ker} h_{2} \circ e_{2}$. Now, for every $R \in \tau$ and $\left(\left(h_{1} \circ e_{1}\right)\left(a_{1}\right), \ldots,\left(h_{1} \circ e_{1}\right)\left(a_{k}\right)\right) \in R^{\widehat{\mathbf{A}}_{1}}$, we have $\left(a_{1}, \ldots, a_{k}\right) \in R^{\mathbf{A}}$, because $h_{1} \circ e_{1}$ preserves the complements of all relations of $\mathbf{A}$ due to Lemma 2. But this implies $\left(\left(h_{2} \circ e_{2}\right)\left(a_{1}\right), \ldots,\left(h_{2} \circ e_{2}\right)\left(a_{k}\right)\right) \in R^{\widehat{\mathbf{A}}_{2}}$, because $h_{2} \circ e_{2}$ is a homomorphism. By Lemma $2, g$ preserves the complements of all relations of $\widehat{\mathbf{A}}_{1}$ and

$$
\operatorname{ker} g=\bigcup\left\{R^{\widehat{\mathbf{A}}_{1}} \mid R \in \tau \text { and } R^{\widehat{\mathbf{A}}_{2}} \subseteq \triangle_{\widehat{A}_{2}}\right\}=\triangle_{\widehat{A}_{1}} .
$$

Hence $g$ is an isomorphism that additionally satisfies $g \circ h_{1} \circ e_{1}=h_{2} \circ e_{2}$. Let $\widehat{\mathbf{B}}_{1}$ and $\widehat{\mathbf{B}}_{2}$ be the substructures of $\mathbf{D}$ on $h_{1}\left(B_{1}\right)$ and $h_{2}\left(B_{2}\right)$, respectively. Now consider the inclusions $\widehat{e}_{i}: \widehat{\mathbf{A}}_{i} \hookrightarrow \widehat{\mathbf{B}}_{i}$. Since Age $(\mathbf{D})$ has AP, there exists 
$\mathbf{C} \in$ Age $(\mathbf{D})$ together with $\widehat{f}_{i}: \widehat{\mathbf{B}}_{i} \hookrightarrow \mathbf{C}$ and $e: \mathbf{C} \hookrightarrow \mathbf{D}$ such that $\widehat{f}_{1} \circ \widehat{e}_{1}=$ $\widehat{f}_{2} \circ \widehat{e}_{2} \circ g$. We define the homomorphisms $f_{i}: \mathbf{B}_{i} \rightarrow \mathbf{D}$ by $f_{i}:=e \circ \widehat{f}_{i} \circ h_{i}$. Then, for every $a \in A$,

$$
\begin{aligned}
f_{1} \circ e_{1}(a) & =e \circ \widehat{f}_{1} \circ h_{1} \circ e_{1}(a)=e \circ \widehat{f}_{1} \circ \widehat{e}_{1} \circ h_{1} \circ e_{1}(a) \\
& =e \circ \widehat{f}_{2} \circ \widehat{e}_{2} \circ g \circ h_{1} \circ e_{1}(a)=e \circ \widehat{f}_{2} \circ \widehat{e}_{2} \circ h_{2} \circ e_{2}(a) \\
& =e \circ \widehat{f}_{2} \circ h_{2} \circ e_{2}(a)=f_{2} \circ e_{2}(a) .
\end{aligned}
$$

Hence $\mathbf{D}$ is a patchwork. For the other direction, suppose that $\mathbf{D}$ is a patchwork. Let $\mathbf{A}, \mathbf{B}_{1}, \mathbf{B}_{2}$ be finite $\tau$-structures with $e_{i}: \mathbf{A} \hookrightarrow \mathbf{B}_{i}$ and $h_{i}: \mathbf{B}_{i} \hookrightarrow \mathbf{D}$. Since $\mathbf{B}_{1}$ and $\mathbf{B}_{2}$ are isomorphic to substructures of $\mathbf{D}$, they are clearly JEPD. Thus, as $\mathbf{D}$ is a patchwork, there exist homomorphisms $f_{i}: \mathbf{B}_{i} \rightarrow \mathbf{D}$ with $f_{1} \circ e_{1}=f_{2} \circ e_{2}$. By Lemma 2, the $f_{i}$ preserve the complements of all relations of $\mathbf{B}_{i}$, and

$$
\operatorname{ker} f_{i}=\bigcup\left\{R^{\mathbf{B}_{i}} \mid R \in \tau \text { and } R^{\mathbf{D}} \subseteq \triangle_{D}\right\}=\operatorname{ker} h_{i}=\triangle_{B_{i}} .
$$

This means that $f_{i}$ are embeddings. We obtain AP for Age (D) by choosing $\mathbf{C}$ to be the substructure of $\mathbf{D}$ on $f_{2}\left(B_{1}\right) \cup f_{1}\left(B_{2}\right)$.

Recall that, to obtain JDJEPD, we actually need to take the $d$-reduct of a given $\omega$-categorical structure, rather than the structure itself. Fortunately, homogeneity transfers from $\mathbf{D}$ to $\mathbf{D}^{\leqslant d}$ (see [8] for the proof).

Lemma 3. Let $\mathbf{D}$ be a countable homogeneous structure with a finite relational signature $\tau$. Then $\mathbf{D}^{\leqslant d}$ is homogeneous for every $d$ that exceeds or is equal to the maximal arity of the symbols from $\tau$.

Finite Boundedness. The only property of $\omega$-admissible structures we have not yet considered in this section is the decidability of the CSP. One possibility to achieve this is to consider finitely bounded structures. For a class $\mathcal{N}$ of $\tau$-structures, we denote by $\operatorname{Forb}_{e}(\mathcal{N})$ the class of all finite $\tau$ structures not embedding any member of $\mathcal{N}$. We say that a structure $\mathbf{A}$ is finitely bounded if its signature is finite and $\operatorname{Age}(\mathbf{A})=\operatorname{Forb}_{e}(\mathcal{N})$ for a finite $\mathcal{N}$ [14]. Note that $\mathbf{A}$ is finitely bounded iff there exists a universal FO sentence $\Phi(\mathbf{A})$ s.t. $\mathbf{B} \in \operatorname{Age}(\mathbf{A})$ iff $\mathbf{B}=\Phi(\mathbf{A})[8]$.

The structure $\mathbf{Q}$ is finitely bounded. To show this, we can use the set $\mathcal{N}$ consisting of the four structures depicted in Fig. 1: the self loop, the 2-cycle, the 3-cycle, and two isolated vertices. We must show that $\operatorname{Age}(\mathbf{Q})=\operatorname{Forb}_{e}(\mathcal{N})$. Clearly, none of the structures in $\mathcal{N}$ embeds into a linear order, which shows Age $(\mathbf{Q}) \subseteq \operatorname{Forb}_{e}(\mathcal{N})$. Conversely, assume that $\mathbf{A}$ is an element of $\operatorname{Forb}_{e}(\mathcal{N})$. We must show that $<^{\mathbf{A}}$ is a linear order. Since $\mathcal{N}$ contains the self loop, we have $(a, a) \notin<^{\mathbf{A}}$ for all $a \in A$, which shows that $<^{\mathbf{A}}$ is irreflexive. For distinct elements $a, b \in A$, we must have $a<^{\mathbf{A}} b$ or $b<^{\mathbf{A}} a$ since otherwise the structure consisting of two isolated vertices could be embedded into $\mathbf{A}$. This shows that any two distinct elements are comparable w.r.t. $<^{\mathbf{A}}$. To show that $<^{\mathbf{A}}$ is transitive, 

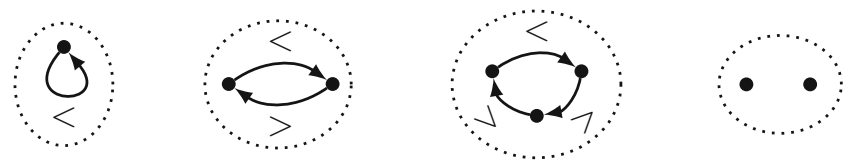

Fig. 1. A set of four forbidden substructures for $\mathbf{Q}=(\mathbb{Q} ;<)$.

assume that $a<^{\mathbf{A}} b$ and $b<^{\mathbf{A}} c$ holds. Since the 2-cycle does not embed into $\mathbf{A}$, $a$ and $c$ must be distinct, and are thus comparable. We cannot have $c<^{\mathbf{A}} a$ since then we could embed the 3-cycle into $\mathbf{A}$. Consequently, we must have $a<^{\mathbf{A}} c$, which proves transitivity. This show that $\mathbf{A}$ is a linear order. As formula $\Phi(\mathbf{Q})$ we can take the conjunction of the usual axioms defining linear orders.

Finitely bounded structures are interesting since their CSP and their firstorder theory are decidable. The first result can, e.g., be found in [13] (Theorem 4) and the second result is stated in $[28,29]$ (see [8] for a detailed proof).

Proposition 4. Let $\mathbf{D}$ be finitely bounded homogeneous structure with $|D|>1$. Then $C S P(\mathbf{D})$ is decidable in NP and the FO theory of $\mathbf{D}$ is PSPACE-complete.

The following proposition, whose proof can be found in [8], implies that Proposition 4 applies not only to a given finitely bounded homogeneous structure $\mathbf{D}$, but also to its $d$-reduct $\mathbf{D}^{\leqslant d}$.

Proposition 5. Let $\mathbf{A}$ be a finitely bounded homogeneous structure and $\mathbf{B}$ a structure with the same domain and finitely many relations that are $F O$ definable in $\mathbf{A}$. Then $\mathbf{B}$ is a reduct of a finitely bounded homogeneous structure.

We are now ready to formulate our first sufficient condition for $\omega$ admissibility.

Theorem 5. Let $\mathbf{D}$ be a finitely bounded homogeneous relational structure with at most $d$-ary relations for some $d \geq 2$. Then $\mathbf{D} \leqslant d$ is $\omega$-admissible.

Proof. It follows directly from its definition that $\mathbf{D}^{\leqslant d}$ is JEPD. Since $d \geq 2$, it is clearly also JD. By Lemma $3, \mathbf{D}^{\leqslant d}$ is homogeneous and $\omega$-categorical. Thus $\mathbf{D}$ has homomorphism compactness by Corollary 1 . By Theorem 4 , Age $\left(\mathbf{D}^{\leqslant d}\right)$ has AP. Thus $\mathbf{D}^{\leqslant d}$ is a patchwork by Proposition 3. By Proposition 5, Lemma 1, and Proposition $4, \operatorname{CSP}\left(\mathbf{D}^{\leqslant d}\right)$ is in NP. Hence $\mathbf{D}^{\leqslant d}$ is $\omega$-admissible.

This theorem, together with Theorem 1, immediately yields decidability for $\mathcal{A L C}_{\mathrm{V}^{+}}^{d}\left(\mathbf{D}^{\leqslant d}\right)$. The following corollary shows that we can even allow for arbitrary FO definable relations with arity bounded by $d$ in the concrete domain.

Corollary 2. Let $\mathbf{D}$ be a finitely bounded homogeneous relational structure with at most $d$-ary relations for some $d \geq 2$. Then concept satisfiability in $\mathcal{A L C}_{F O}^{d}(\mathbf{D})$ w.r.t. TBoxes is decidable. 
The idea for proving this result is to reduce concept satisfiability in $\mathcal{A L C}_{\text {FO }}^{d}(\mathbf{D})$ to concept satisfiability in $\mathcal{A L C}_{\mathrm{V}^{+}}^{d}\left(\mathbf{D}^{\leqslant d}\right)$. We know that every at most $d$-ary relation over $D$ FO definable in $\mathbf{D}$ can be obtained as a disjunction of atomic formulas built using the signature of $\mathbf{D}^{\leqslant d}$. What still needs to be shown is that, given a first-order formula in the signature of $\mathbf{D}$ with at most $d$ free variables, this disjunction can effectively be computed (see [8] for how this can be proved).

Cores. Finally, we consider the situation where we have a homogeneous relational structure $\mathbf{D}$ with finitely many at most $d$-ary relations that is not finitely bounded, but which we know (by some other means) to have a decidable CSP. In this situation, we can show decidability for $\mathcal{A L C}_{\exists^{+}}^{d}(\mathbf{D})$ under one additional assumption. A structure $\mathbf{D}$ is called a core if every endomorphism from $\mathbf{D}$ to itself is an embedding. It was shown in [9] that, if $\mathbf{D}$ is a homogeneous core, then the orbits of tuples over $D$ under $\operatorname{Aut}(\mathbf{D})$ are pp definable in $\mathbf{D}$. As an easy consequence of this result, we obtain our second sufficient condition for $\omega$-admissibility (see [8] for the proof).

Theorem 6. Let $\mathbf{D}$ be a homogeneous relational structure with finitely many at most $d$-ary relations for some $d \geq 2$ that is a core and has a decidable CSP. Then $\mathbf{D}^{\leqslant d}$ is $\omega$-admissible.

By showing that concept satisfiability in $\mathcal{A L C}_{\exists+}^{d}(\mathbf{D})$ can effectively be reduced to concept satisfiability in $\mathcal{A L C}_{\mathrm{V}^{+}}^{d}\left(\mathbf{D}^{\leqslant d}\right)$, we obtain the following decidability result (see [8] for the proof).

Corollary 3. Let $\mathbf{D}$ be a homogeneous relational structure with finitely many at most d-ary relations for some $d \geq 2$ that is a core and has a decidable CSP. Then concept satisfiability in $\mathcal{A L C}_{\exists+}^{d-}(\mathbf{D})$ w.r.t. TBoxes is decidable.

\section{Application and Discussion}

In this section, we discuss how the results of Sect. 4 can be used to obtain specific $\omega$-admissible concrete domains. But let us first start with a caveat.

Finiteness of Signature Matters. In Corollary 2 and Corollary 3, the signature of the structure $\mathbf{D}$ is required to be finite. This restriction is needed to obtain decidability. For instance, the expansion of the structure $\left(\mathbb{Z} ;+_{1}\right)$ from Sect. 3.1 by all relations $+_{k}=\left\{(m, n) \in \mathbb{Z}^{2} \mid m+k=n\right\}$ for $k \in \mathbb{Z}$ is homogeneous, and satisfiability of finite conjunctions of constraints is decidable in this structure. However, we have seen in Proposition 1 that reasoning with $\left(\mathbb{Z} ;+_{1}\right)$ as a concrete domain w.r.t. TBoxes is undecidable.

(Un) decidability of the Conditions. If one intends to use Theorem 5 to obtain an $\omega$-admissible concrete domain, one could start with selecting a finite set $\mathcal{N}$ of bounds, i.e., forbidden $\tau$-substructures, for a finite signature $\tau$. The question is then whether $\mathcal{N}$ really induces a finitely bounded structure, i.e., whether 
there is a $\tau$-structure $\mathbf{D}$ such that Age $(\mathbf{D})=\operatorname{Forb}_{e}(\mathcal{N})$. This question is in general undecidable. In fact, it is shown in [17] that the joint embedding property $(J E P)$ is undecidable for classes of structures that are definable by finitely many bounds. In addition, it is known that a class of structures definable by finitely many bounds has JEP iff this class is the age of some countably infinite structure (see [25], Theorem 6.1.1). However, if one restricts the attention to binary signatures, then it is decidable whether a class of the form $\operatorname{Forb}_{e}(\mathcal{N})$ has AP [12]. If this is the case, then the Fraïssé limit $\mathbf{D}$ of $\operatorname{Forb}_{e}(\mathcal{N})$ is a finitely bounded homogeneous structure satisfying $\operatorname{Age}(\mathbf{D})=\operatorname{Forb}_{e}(\mathcal{N})$ by Theorem 4 .

Reproducing Known Results. The examples for $\omega$-admissible concrete domains given in [33] were RCC8 and Allen's interval algebra, for which the patchwork property is proved "by hand" in [33]. Given Theorem 5, we obtain these results as a consequence of known results from model theory. It was shown in [15] that RCC8 has a representation by a homogeneous structure $\mathbf{R}$ with a finite relational signature (Theorem 2 in [15]). Since Age $(\mathbf{R})$ has a finite universal axiomatization (Definition 3 in [15]), $\mathbf{R}$ is finitely bounded. For Allen's interval algebra, it was shown in [24] that it has a representation by a homogeneous structure $\mathbf{A}$ with a finite relational signature. Since Age $(\mathbf{A})$ has a finite universal axiomatization, $\mathbf{A}$ is finitely bounded. Our running example $\mathbf{Q}=(\mathbb{Q},<)$ also satisfies the preconditions of Theorem 5, and thus Corollary 2 yields decidability of $\mathcal{A L C}_{\mathrm{FO}}^{d}(\mathbf{Q})$ with TBoxes. For $\mathbf{Q}$ extended just with $>, \leq, \geq,=, \neq$, decidability was proved in [30], using an automata-based procedure. Our results show that there is also a tableau-based decision procedure for this logic.

Expansions, Disjoint Unions, and Products. When modelling concepts in a DL with concrete domain $\mathbf{D}$, it is often useful to be able to refer to specific elements $d$ of the domain, i.e., to have unary predicate symbols $=_{d}$ that are interpreted as $\{d\}$. We can show that the class of reducts of finitely bounded homogeneous structures is closed under expansion by finitely many such relations [8].

It would also be useful to be able to refer to predicates of different concrete domains (say RCC8 and Allen) when defining concepts. In [5], it was shown that admissible concrete domains are closed under disjoint union. We can prove the corresponding result for finitely bounded homogeneous structures [8]. Using disjoint union to refer to several concrete domain works well if the paths employed in concrete domain constructors contain only functional roles, which is the case considered in [5]. However, if we allow for non-functional roles in paths, then using disjoint union is not appropriate. In general, if $R, R^{\prime}$ are two binary relations over $D$ and $r \in \mathrm{N}_{\mathrm{R}} \backslash \mathrm{N}_{\mathrm{fR}}$, then the situation where an individual $x$ has an $r$-successor $y$ with features related through both $R$ and $R^{\prime}$ cannot be described using the disjoint union of $(D ; R)$ and $\left(D ; R^{\prime}\right)$ as a concrete domain (see [8] for details).

To overcome this problem, we propose to use the so-called full product [10]. Let $\mathbf{A}_{1}, \ldots, \mathbf{A}_{k}$ be finitely many structures with disjoint relational signatures $\tau_{1}, \ldots, \tau_{k}$. The full product of $\mathbf{A}_{1}, \ldots, \mathbf{A}_{k}$, denoted by $\mathbf{A}_{1} \otimes \cdots \otimes \mathbf{A}_{k}$, has as its domain the Cartesian product $A:=A_{1} \times \cdots \times A_{k}$ and as its signature the 
union of the signatures $\tau_{i}$. For $a \in A_{1} \times \cdots \times A_{k}$ and $i \in[k]$, we denote the $i$ th component of the tuple $a$ by $a[i]$. The relations are defined as follows:

$$
R^{\mathbf{A}_{1} \times \cdots \times \mathbf{A}_{k}}:=\left\{\left(a_{1}, \ldots, a_{n}\right) \in A^{n} \mid\left(a_{1}[i], \ldots, a_{n}[i]\right) \in R^{\mathbf{A}_{i}}\right\}
$$

for every $i \in[k]$ and every $n$-ary $R \in \tau_{i}$. We show in [8] that the full product preserves homogeneity and finite boundedness, and thus the prerequisites for Theorem 5 and Corollary 2 are preserved under building the full product.

Proposition 6. Let $\mathbf{A}_{1}, \ldots, \mathbf{A}_{k}$ be finitely bounded homogeneous structures with disjoint relational signatures $\tau_{1}, \ldots, \tau_{k}$ such that, for $i \in[k], \tau_{i}$ contains the symbol $={ }_{i}$, which is defined in $\mathbf{A}_{i}$ as $\triangle_{A_{i}}$. Then $\mathbf{A}_{1} \otimes \cdots \otimes \mathbf{A}_{k}$ is a finitely bounded homogeneous structure.

Together with Proposition 4 this also yields a general complexity result for combinations of constraints over several finitely bounded homogeneous templates. Such combinations were previously considered in the literature in special cases; for example, for RCC8 and Allen [21].

Henson Digraphs. A directed graph is a tournament if every two distinct vertices in it are connected by exactly one directed edge. In [23], Henson proved that there are uncountably many homogeneous directed graphs by showing that, for any set $\mathcal{N}$ of finite tournaments (plus the loop and the 2 -cycle) such that no member of $\mathcal{N}$ is embeddable into any other member of $\mathcal{N}, \operatorname{Forb}_{e}(\mathcal{N})$ is an amalgamation class whose Fraïssé limit is a homogeneous directed graph. Furthermore, the Fraïssé limits for two distinct sets of such tournaments are distinct as well. In the literature, such directed graphs are often called Henson digraphs [34]. If $\mathbf{G}$ is a Henson digraph, then $\operatorname{Age}(\mathbf{G})=\operatorname{CSP}(\mathbf{G}) .{ }^{5}$ Clearly, only countably many Henson digraphs can have a decidable CSP. Beside the finitely bounded ones (see Proposition 4), there is an interesting example constructed using the infinite set of non-isomorphic tournaments from Henson's original proof of uncountability. Consider the tournaments $\mathbf{T}_{1}, \mathbf{T}_{2}, \ldots$ with domains [2], [3], .. such that the edge relation of $\mathbf{T}_{n}$ consists of the edges $(i, j)$ for every $j=i+1$ with $0 \leq i \leq n$, $(0, n+1)$, and $(j, i)$ for every $j>i+1$ with $(i, j) \neq(0, n+1)$. It was shown in [11] that the CSP of the Henson digraph corresponding to $\mathcal{N}:=\left\{\mathbf{T}_{1}, \mathbf{T}_{2}, \ldots\right\}$ is CONP-complete. This digraph is a homogeneous core, and its CSP is decidable. Thus, it satisfies the requirements of Corollary 3. However, it is clearly not finitely bounded, and thus does not satisfy the requirements of Corollary 2. Conversely, it is known that the random graph is finitely bounded and homogeneous [25], but it is not a core [9]. This shows that the class of structures covered by Corollary 3 is incomparable with the one covered by Corollary 2 .

\section{Conclusion}

We have shown that $\omega$-admissibility, which was introduced in the DL community to obtain decidable extensions of DLs by concrete domains, is closely related to

\footnotetext{
${ }^{5}$ One direction is obvious, the other holds because homomorphisms between directed graphs cannot contract any edges.
} 
well-known notions from model theory. Given the fact that a large number of homogeneous structures are known from the literature [34] and that homogeneous and finitely bounded structures play an important rôle in the CSP community, we believe that our work will turn out to be useful for locating new $\omega$-admissible concrete domains.

This is not the first model-theoretic description of a sufficient condition for decidability of reasoning in DLs with concrete domains in the presence of TBoxes. The existence of homomorphism is definable (EHD) property was used in [19] to obtain decidability results for DLs with concrete domains. However, the way the concrete domain is integrated into the DL in [19] is different from the classical one employed by us and used in all other papers on DLs with concrete domains. In [19], constraints are always placed along a linear path stemming from a single individual, which is rather similar to the use of constraints in temporal logics $[18,20]$. In contrast, in the classical setting of DLs with concrete domains, one can compare feature values of siblings of an individual.

\section{References}

1. Allen, J.F.: Maintaining knowledge about temporal intervals. Commun. ACM 26(11), 832-843 (1983)

2. Baader, F., Bürckert, H.J., Hollunder, B., Nutt, W., Siekmann, J.H.: Concept logics. In: Lloyd, J.W. (ed.) Computational Logics. ESPRIT Basic Research Series, pp. 177-201. Springer, Heidelberg (1990). https://doi.org/10.1007/978-3-642-76274$1 \_10$

3. Baader, F., Calvanese, D., McGuinness, D., Nardi, D., Patel-Schneider, P.F. (eds.): The Description Logic Handbook: Theory, Implementation, and Applications. Cambridge University Press, Cambridge (2003)

4. Baader, F., Hanschke, P.: A schema for integrating concrete domains into concept languages. In: Proceedings of the 12th International Joint Conference on Artificial Intelligence (IJCAI 1991), pp. 452-457 (1991). Long version available as [5]

5. Baader, F., Hanschke, P.: A scheme for integrating concrete domains into concept languages. Technical report RR-91-10, Deutsches Forschungszentrum für Künstliche Intelligenz (DFKI) (1991). https://lat.inf.tu-dresden.de/research/ reports/1991/DFKI-RR-91-10.pdf

6. Baader, F., Hanschke, P.: Extensions of concept languages for a mechanical engineering application. In: Jürgen Ohlbach, H. (ed.) GWAI 1992. LNCS, vol. 671, pp. 132-143. Springer, Heidelberg (1993). https://doi.org/10.1007/BFb0018999

7. Baader, F., Horrocks, I., Lutz, C., Sattler, U.: An Introduction to Description Logic. Cambridge University Press, Cambridge (2017)

8. Baader, F., Rydval, J.: Using model-theory to find $\omega$-admissible concrete domains. LTCS-Report 20-01, Chair of Automata Theory, Institute of Theoretical Computer Science, Technische Universität Dresden, Dresden, Germany (2020). https://tudresden.de/inf/lat/reports\#BaRy-LTCS-20-01

9. Bodirsky, M.: The core of a countably categorical structure. In: Diekert, V., Durand, B. (eds.) STACS 2005. LNCS, vol. 3404, pp. 110-120. Springer, Heidelberg (2005). https://doi.org/10.1007/978-3-540-31856-9_9

10. Bodirsky, M.: Complexity classification in infinite-domain constraint satisfaction. Habilitation thesis, Université Diderot - Paris 7 (2012). https://arxiv.org/pdf/ 1201.0856.pdf 
11. Bodirsky, M., Grohe, M.: Non-dichotomies in constraint satisfaction complexity. In: Aceto, L., Damgård, I., Goldberg, L.A., Halldórsson, M.M., Ingólfsdóttir, A., Walukiewicz, I. (eds.) ICALP 2008. LNCS, vol. 5126, pp. 184-196. Springer, Heidelberg (2008). https://doi.org/10.1007/978-3-540-70583-3_16

12. Bodirsky, M., Knäuer, S., Starke, F.: AMSNP: a tame fragment of existential second-order logic. In: Proceedings of the 16th Conference on Computability in Europe - Beyond the Horizon of Computability (CiE 2020). Lecture Notes in Computer Science. Springer (2020). https://arxiv.org/abs/2001.08190.pdf

13. Bodirsky, M., Mottet, A.: Reducts of finitely bounded homogeneous structures, and lifting tractability from finite-domain constraint satisfaction. In: Proceedings of the 31st Annual ACM/IEEE Symposium on Logic in Computer Science (LICS 2016), pp. 623-632. ACM/IEEE (2016)

14. Bodirsky, M., Nešetřil, J.: Constraint satisfaction with countable homogeneous templates. J. Logic Comput. 16(3), 359-373 (2006)

15. Bodirsky, M., Wölfl, S.: RCC8 is polynomial on networks of bounded treewidth. In: Proceedings of the 22nd International Joint Conference on Artificial Intelligence (IJCAI 2011). IJCAI/AAAI (2011)

16. Bojańczyk, M., Segoufin, L., Toruńczyk, S.: Verification of database-driven systems via amalgamation. In: Proceedings of the 32nd ACM SIGMOD-SIGACT-SIGART Symposium on Principles of Database Systems (PODS 2013), pp. 63-74. ACM (2013)

17. Braunfeld, S.: The undecidability of joint embedding and joint homomorphism for hereditary graph classes. Discrete Math. Theoret. Comput. Sci. 21(2) (2019). https://arxiv.org/pdf/1903.11932.pdf

18. Carapelle, C., Feng, S., Kartzow, A., Lohrey, M.: Satisfiability of ECTL* with local tree constraints. Theory Comput. Syst. 61(2), 689-720 (2017). https://doi.org/10. 1007/s00224-016-9724-y

19. Carapelle, C., Turhan, A.Y.: Description logics reasoning wrt general TBoxes is decidable for concrete domains with the EHD-property. In: Proceedings of the 22nd European Conference on Artificial Intelligence (ECAI 2016), pp. 1440-1448. IOS Press (2016)

20. Demri, S., D'Souza, D.: An automata-theoretic approach to constraint LTL. Inf. Comput. 205(3), 380-415 (2007)

21. Gerevini, A., Nebel, B.: Qualitative spatio-temporal reasoning with RCC-8 and Allen's interval calculus: Computational complexity. In: Proceedings of the 15th European Conference on Artificial Intelligence (ECAI 2002), pp. 312-316. IOS Press (2002)

22. Haarslev, V., Möller, R., Wessel, M.: The description logic $\mathcal{A} \mathcal{L C N} \mathcal{H}_{R+}$ extended with concrete domains: a practically motivated approach. In: Goré, R., Leitsch, A., Nipkow, T. (eds.) IJCAR 2001. LNCS, vol. 2083, pp. 29-44. Springer, Heidelberg (2001). https://doi.org/10.1007/3-540-45744-5_4

23. Henson, C.W.: Countable homogeneous relational structures and $\aleph_{0}$-categorical theories. J. Symb. Logic 37(3), 494-500 (1972)

24. Hirsch, R.: Relation algebras of intervals. Artif. Intell. 83(2), 267-295 (1996)

25. Hodges, W.: A Shorter Model Theory. Cambridge University Press, Cambridge (1997)

26. Hoehndorf, R., Schofield, P.N., Gkoutos, G.V.: The role of ontologies in biological and biomedical research: a functional perspective. Brief. Bioinform. 16(6), 1069$1080(2015)$

27. Horrocks, I., Patel-Schneider, P.F., van Harmelen, F.: From SHIQ and RDF to OWL: the making of a web ontology language. J. Web Semant. 1(1), 7-26 (2003) 
28. Klin, B., Lasota, S., Ochremiak, J., Torunczyk, S.: Homomorphism problems for first-order definable structures. In: 36th IARCS Annual Conference on Foundations of Software Technology and Theoretical Computer Science (FSTTCS 2016). Schloss Dagstuhl-Leibniz-Zentrum für Informatik (2016)

29. Kopczyński, E., Toruńczyk, S.: LOIS: an application of SMT solvers. In: King, T., Piskac, R. (eds.) Proceedings of the 14th International Workshop on Satisfiability Modulo Theories (SMT@IJCAR 2016). CEUR Workshop Proceedings, vol. 1617, pp. 51-60. CEUR-WS.org (2016)

30. Lutz, C.: Interval-based temporal reasoning with general TBoxes. In: Nebel, B. (ed.) Proceedings of the 17th International Joint Conference on Artificial Intelligence (IJCAI 2001), pp. 89-94. Morgan Kaufmann, San Mateo (2001)

31. Lutz, C.: NExpTime-complete description logics with concrete domains. In: Goré, R., Leitsch, A., Nipkow, T. (eds.) IJCAR 2001. LNCS, vol. 2083, pp. 45-60. Springer, Heidelberg (2001). https://doi.org/10.1007/3-540-45744-5_5

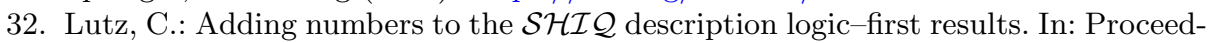
ings of the 8th International Conference on Principles of Knowledge Representation and Reasoning (KR 2002), pp. 191-202. Morgan Kaufmann, Los Altos (2002)

33. Lutz, C., Milicic, M.: A tableau algorithm for description logics with concrete domains and general TBoxes. J. Autom. Reason. 38(1-3), 227-259 (2007). https:// doi.org/10.1007/s10817-006-9049-7

34. Macpherson, D.: A survey of homogeneous structures. Discrete Math. 311(15), 1599-1634 (2011)

35. Minsky, M.L.: Computation: Finite and Infinite Machines. Prentice-Hall, Englewood Cliffs (1967)

36. Pan, J.Z., Horrocks, I.: Reasoning in the $\mathcal{S H O} \mathcal{Q}\left(D_{n}\right)$ description logic. In: Horrocks, I., Tessaris, S. (eds.) Proceedings of the 2002 Description Logic Workshop (DL 2002). CEUR Workshop Proceedings, vol. 53. CEUR-WS.org (2002)

37. Randell, D.A., Cui, Z., Cohn, A.G.: A spatial logic based on regions and connection. In: Proceedings of the 3rd International Conference on the Principles of Knowledge Representation and Reasoning (KR 1992), pp. 165-176. Morgan Kaufmann, Los Altos (1992)

38. Schild, K.: A correspondence theory for terminological logics: preliminary report. In: Mylopoulos, J., Reiter, R. (eds.) Proceedings of the 12th International Joint Conference on Artificial Intelligence (IJCAI 1991), pp. 466-471. Morgan Kaufmann (1991) 